\title{
Managing First Response Medical Aids With An Altruistic Web Application
}

\author{
Stefano Ferretti, Silvia Mirri, Marco Roccetti, Claudio Sermenghi, Vito Conforti \\ Department of Computer Science, University of Bologna \\ Mura Anteo Zamboni 7 \\ 40127, Bologna, Italy \\ \{sferrett, mirri, roccetti, sermengh, vconfort\}@cs.unibo.it
}

\begin{abstract}
The use of the Web as a mean to express and satisfy users' personal needs is widespread. However, this novel way to exploit online applications to give voice to any opinion has rapidly degenerated; now, the Web $\mathbf{2 . 0}$ is more and more recognized as a tool to foster "human egocentrism". Nevertheless, many experts assert that contents produced and distributed through Web 2.0 technologies can be exploited also for "altruistic" purposes. In this paper, we show how contents created through a selfish use of the Web 2.0 can be recycled into "altruistic" applications for pervasive healthcare services and first response medical aids. The idea is that of trading the online advertisement of doctors and healthcare workers with their availability to be located and their agreement to intervene in case of emergency. The key to technically build such pervasive applications is based on an approach to profile patients, doctors and their location, employed together with communication protocols able to spread information over heterogeneous networks.
\end{abstract}

Keywords: Altruistic Web, Pervasive Healthcare, Semantic Data Dissemination, Profiling.

\section{INTRODUCTION}

It is a fact that the umbrella of technologies grouped under the name of Web 2.0 has changed the structure of our society. Thanks to this new paradigm of management and distribution of data, new spaces of freedom are available which enable people to interact, communicate, express their opinion and collaborate inside cyber-communities.

Unfortunately, this positive imagery is partially distorted. The vision of Web 2.0 as a tool for the individual ego, rather than for the entire society, is getting a foothold. The vast potential of Web 2.0 is dissipated in expressions of selfishness, where communities are moved for personal interests like monetary enrichment and self-celebration, without any practical advantage shared with the rest of the society. MySpace, Facebook and blogs are generally mentioned as pure narcissistic services exploited only to gain some kind of visibility [12].

Actually, the dissemination of personal information and advertisements are not, per se, negative aspects. Conversely, several experts are convinced that even a selfish use of Web 2.0 can be recycled for "altruistic" purposes $[4,10,12]$. The idea is that data produced by users to achieve personal objectives or to have fun, can be rerouted and employed in different contexts that may be of help for other people.

In this paper, we show that an altruistic use of data (and resources) produced (exploited) for selfish purposes can be employed for the provision of ubiquitous healthcare services. The aim is to surpass the traditional (and surely already useful) remote monitoring of sick people, usually accomplished at home by exploiting wired communication networks [1]. This can be done via the adoption of wireless-enabled, wearable healthcare monitoring devices that can follow the patient during his/her everyday activities.

To better understand the scenario we have in mind, think of a remotely monitored patient who experiences a sudden and unexpected worsening of her/his health conditions while walking in a park. The healthcare monitoring device could be made able to use some kind of cellular network to directly send an alert message to a first aid station (this is a classic approach where nothing altruistic happens).

However, for many reasons the use of a cellular network cannot be available (or not even the more efficient solution). Hence, alternatively, some ad-hoc wireless network could be exploited so that the alert message produced by the healthcare monitoring device can be propagated till reaching some access point that can route the message toward the first aid station. In this case, resources selfishly used by passers-by can be utilized to create an "altruistic" network exploited to forward such an alert. Therefore, paradoxically, a runner who goes jogging for personal interest becomes an altruistic subject by putting on hand her/his fancy technological resources (e.g., the communication capabilities of her/his iPhone).

But, in any case, this can be not enough. The arrival of doctors called by the remote monitoring station could be not timely. Instead, it would be more effective to propagate the aidrequest until reaching, for instance, the smart phone of a doctor in the neighborhood, who could promptly take action while waiting for the ambulance. The discovery of the doctor can be made via the processing of advertisements that he/she previously selfishly disseminated in the ad-hoc network. In this case, there is a trade between the (selfish) online advertisement of doctors in the area of interest and their 
agreement/availability to be located and to intervene in case of emergency.

The purpose of this paper is that of describing which kinds of technological solutions must be adopted and merged to put this vision into reality. Thus, a logical architecture of the proposed system is delineated (see Section II). Then, patients and healthcare workers (i.e., those which are involved in some form of employment related to healthcare services) must be adequately profiled so as to allow the discovery and matching of experts nearby patients that can be of help in an emergency scenario. We will show that XML languages already exist that can be employed for this purpose (see Section III). Finally, we provide some concluding remarks (see Section IV).

\section{AN AlTRUISTIC WEB APPLICATION FOR FIRST RESPONSE MEDICAL AIDS}

To support ubiquitous healthcare services and allow an altruistic use of resources for the dissemination of data and discovery of medical services, we have devised a Web 2.0 application. The application works as an aggregator of resources and contents that can be combined through public interfaces, feeds, and Web services [4]. The healthcare service will be built on top of such application. The stack of functionalities offered by the application is depicted in Figure 1. All these functionalities are needed for an altruistic use of resources, a pervasive dissemination of data and the realization of ubiquitous healthcare services.

\section{Ubiquitous Healthcare Services}

\section{Filtering \& Discovery}

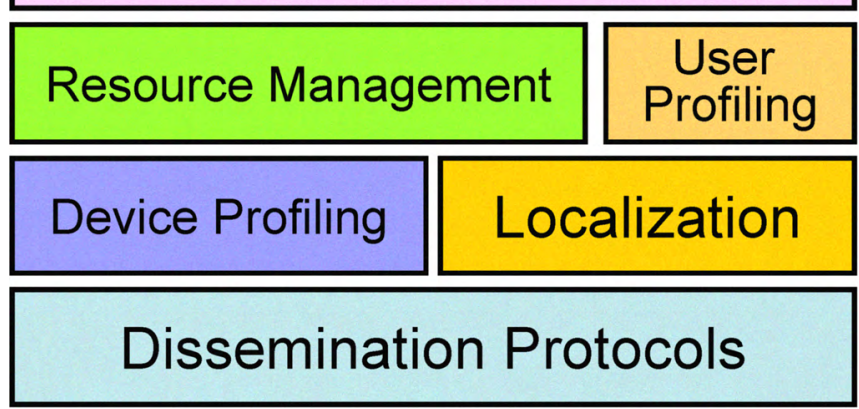

Figure 1. Logical Architecture.

Starting from the bottom, the dissemination of data in heterogeneous wireless networks can be accomplished by resorting one among the plethora of broadcasting schemes devised for ad-hoc nets such as, for example, [11]. Data and services offered by available devices can be discovered only if filtering and profiling mechanisms are exploited, which are able to characterize resources and requests, and perform a match among these. As to resource profiling, CC/PP is a standard to profile all (communication, computation, healthcare) devices [13]. Then, localization of users and resources in general is necessary to support the kind of services we have in mind. Several technologies and services exist that provide such functionalities, e.g., GPS, RFID, localization through radio waves, as well as XML based technologies such as GPX [7]. This allows for a proper management (and donation) of resources.

Concurrently, also users must be profiled and located. We discuss in the next Section our proposal for profiling and locating patients and healthcare workers, specifically thought for healthcare services. It relies on XML based technologies. On top of these services, filtering and discovery of resources, data, services and humans can be accomplished.

Interestingly, the development of an altruistic application could be made easier by resorting to services which have been expressively devised for the support of selfish applications, e.g., specific services being devised to disseminate advertisements. For instance, Ad-Torrent is a digital billboard system that offers location-based advertisement messages (named $A d s$ ), guided by a particular keyword search [9]. It provides means to search and filter queries or contents. Based on Ad-Torrent, each entity in a certain area can generate Ads (which basically consist in associating keywords and metadata to that entity) and disseminate them via the Ad-Torrent system. Potential customers in the neighborhood will receive these Ads if their profiles match those of the Ads. Clearly, potential customers have to subscribe the Ad-Torrent application providing keywords and tags that identify their profiles (i.e., interests, description of their electronic equipments, working experience, etc.). Ad-Torrent is hence an example of system that can be exploited to discover and filter data, human and resources.

We now better explain how healthcare monitoring and first response aid services can be built on top of such architecture. We assume that users subscribe to the platform, because of the services that provides or just because people know that by joining it they might help somebody else. Upon subscription, the system profiles users and devices. These profiles are provided for each of the resources that are made available to be classified and categorized through tags to facilitate their storage.

Certainly, the user with any forms of healthcare issues will subscribe to the system (besides being something altruistic, it could be of help also for him/her in finding quicker aid in case of necessity). Moreover, it is likely that also the doctors and healthcare workers in general will subscribe to the service. They could selfishly join the service just to advertise his/her medical specialization through his/her smart-phone and find new customers in a very cheap way. They are hence motivated in providing all details for their profiles to join the service and to set up their smart phones (or other kinds of communication devices) so as to make them able to diffuse their Ads around. At the same time, such devices are now identified by the system and can be retrieved by to implement altruistic services.

Resources are then altruistically employed as virtual elements to be part of the ubiquitous healthcare service. Thus, thanks to this application, communication capabilities of users spread around a certain area can be exploited in order to propagate messages. For example, through Bluetooth a cell phone may interact with a PDA which has also installed a WiFi network card. It can thus connect to other Wi-Fi enabled 
terminals till reaching, hop by hop, the destination or an access point that can route the message to the Internet.

Such a scheme can be exploited to offer various healthcare services. It represents an important strategy to disseminate alert messages which represent a request for help. This way, the message can be propagated till reaching a healthcare worker accidentally located nearby the user asking for help, as well as the nearest first aid station. When the doctor's phone is reached by the aid-request message from the sick person, it rings and loads a Google Map showing him his position, the sick person's position, and information about the problem.

However, other application scenarios are possible. For instance, in case of an emergency, the collaborative use of resources and devices may be exploited to activate some camera hold by a user (tagged by the system at subscription time) to transmit a video stream. This could be of real help, since first responders may gain from the video an idea of the real situation after an accident and prepare to take action in the proper way.

\section{PROFILING DOCTORS AND PATIENTS FOR A FAST RESPONSE}

Focusing on the specific technologies employed for the provision of pervasive healthcare services, there are three main factors that need to be considered. First, how to profile a given patient and his/her clinical issues. This is of main importance to ensure that, in case of some sudden attack, the alert message to be disseminated can give some information to the first aid station or to look for a specialized doctor in the neighborhood. This allows, for instance, to ask for a cardiologist when the patient had some heart disease in the past. Second, it is important to profile the doctor, and in general all healthcare workers, to allow a proper matching during the discovery phase. Third, it is important to identify the location where users are placed at a given instant. All these issues are discussed in the next, together with a proposal to face and merge them all, thus guaranteeing pervasive healthcare services.

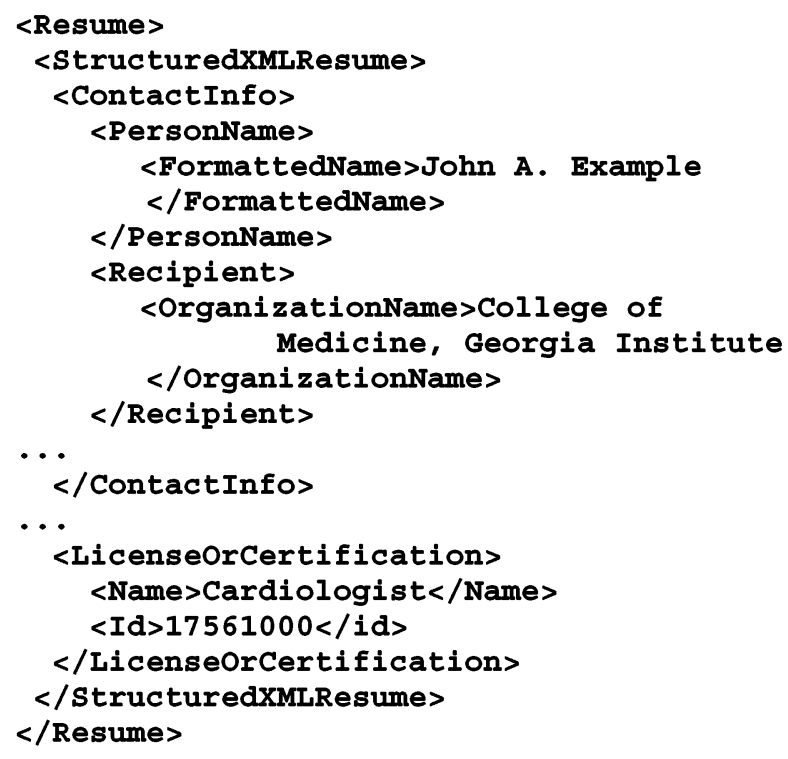

Several XML-based technologies have been developed with the purpose of creating databases containing doctors, patients, diseases and adopted treatments. For instance, doctors can be profiled through the HR-XML [6]. Such specification allows characterizing any clinician based on his/her personal information, organization, medical specialization etc. The XML code reported in Figure 2 is an example of such specification. The code reports information of a hypothetical cardiologist named "John A. Example". The code also reports information such as the organization he belongs to and other data (not reported here).

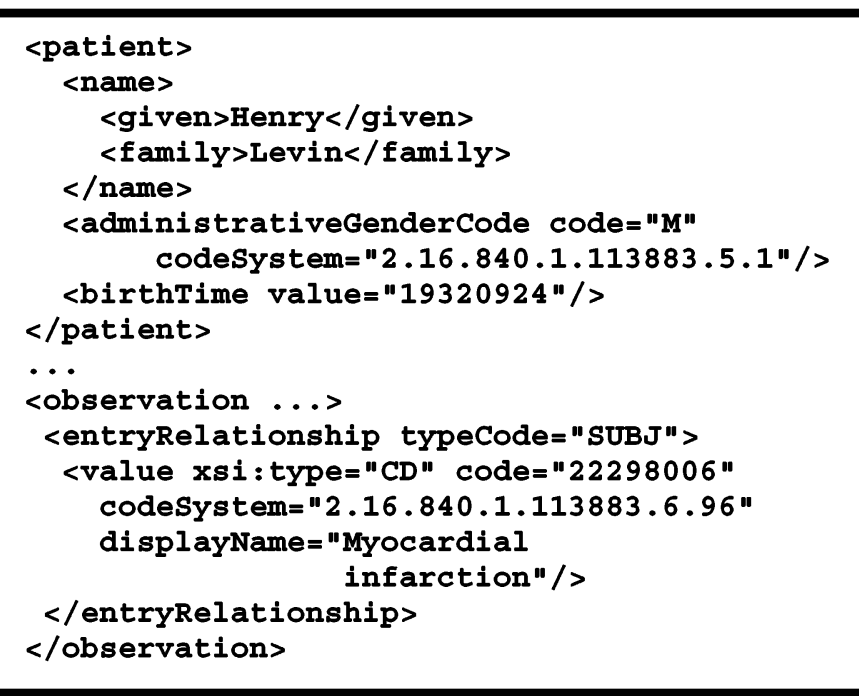

Figure 3. Example of CCD code.

The interesting aspect here is that each medical specialization has its own unique identifier. Thus, in principle any database reporting tuples of this kind can be queried to retrieve information and discover doctors and clinicians corresponding to a given specialization.

On the other hand, a patient can be characterized using the HITSP's (Healthcare Information Technology Standards Panel) CCD (Continuity of Care Document), a health data information contained within a personal health record for the purpose of information exchange [2]. The idea is that, based on this specification, any healthcare worker can record patient information in a consistent manner. The XML code associated to this profile specifies general details of the patient, as well as his/her medical problems he/she had. Figure 3 reports an example of such CCD code, where a patient is characterized. According to the example, the patient's name is "Henry Levin" and in the code it is stated that in the past he had a myocardial infarction (through the CCD specification, it is also possible to report the exact data, not reported here for the sake of simplicity).

An important aspect to mention is that each type of disease has an unique numerical identifier. But most important, similar diseases have near numerical code representations (sharing the same prefix), thus forming a sort of network where neighbour ids correspond to diseases that can be monitored and controlled by the same medical expert.

Figure 2. Example of HR-XML code. 
Finally, GPX (the GPS Exchange Format) is a light-weight XML data format for the interchange of GPS data between applications and Web services on the Internet [7]. Again, an example of the XML code related to this specification is given in Figure 4. Specifically, the two attributes lat and lon are worth of mention which specify the latitude and the longitude, respectively.

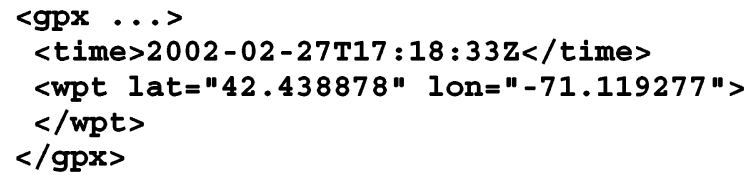

Figure 4. Example of GPX code

By exploiting these specifications, a discovery mechanism which practically allows developing the application is as follows. Healthcare workers are profiled using HR-XML. Upon voluntarily recruitment, such profile is kept by the clinician in his/her mobile device while he/she is doing his/her own daily activities. The profile can be disseminated using different distribution networks, such as the mentioned Ad-Torrent [9], as well as publish-subscribe systems, or general peer-to-peer DHT networks [3, 14]. The idea is that Ad-Torrent should be the primary meta-data dissemination mechanism. Indeed, potential patients in the neighbourhood of a clinician may receive advertisements if their profiles match those of the ads, based on an ad-hoc communication paradigm. Moreover, the use of a static publish-subscribe system can be exploited as an alternative solution to disseminate metadata and perform filtering. Obviously, the information to be distributed based on such approach must refer to the clinical data and the geographical area where the user is (which is instead implicit in an ad-hoc network).

Based on this kind of approaches, patients' devices can be made able to spread first aid requests. Of course, in case of attack or health complications, it would be appropriate if the patient could be first helped by some medical specialist, which is an expert with respect to the diseases previously suffered by the patient. In substance, a match is required between the various available healthcare workers which are nearby the user, and the clinical issues contained in the user profile.

A ranking must be performed based on some metric which includes both the similarity between the specialization of the clinician and the disease, and also the proximity between the two users. A mapping disease/specialization must thus be performed, employed together with similarity algorithms. This is however a simple task to do, since diseases of the same kind share the same prefixes.

Needless to say, it is also important that all the employed technological means and information can be exploited without causing the unwanted diffusion of personal data of the patient. Thus during the alert spreading, security and anonymity mechanisms are needed that guarantee that only the clinical issues are (encrypted,) disseminated and read only by those who are allowed, i.e., healthcare workers.

\section{CONCLUSIONS}

We have presented the design of an altruistic application devised to support ubiquitous healthcare services. Dissemination of data can be accomplished by resorting to unused resources of users which accept to participate and being part of the community. Then, patients and medical staff need to be adequately profiled in order to allow the discovery of healthcare workers and performing a match between the clinical issues of patients and the expertise of the clinicians.

In this work, we have outlined how XML technologies can be exploited to discover clinicians nearby patients who need some help. We have also proposed the use of a selfish application, i.e., Ad-Torrent, as the service for distributing metadata. This confirms our claim that technologies can be reused and exploited for promoting the development of new altruistic applications.

\section{REFERENCES}

[1] _, "Remote Monitoring Saves Lives Of Heart Patients, Research Shows", ScienceDaily, April 2007, available at http://www.sciencedaily.com/releases/2007/04/070420133738.htm, last visited December 2008.

[2] Continuity of Care Document (CCD), web site: http://www.hitsp.org/ConstructSet_Details.aspx?\&PrefixAlpha=4\&Prefi $\mathrm{xNumeric}=32$, last visited December 2008.

[3] Eugster, P. T., Felber, P. A., Guerraoui, R., and Kermarrec, A. 2003. The many faces of publish/subscribe. ACM Comput. Surv. 35, 2 (Jun. 2003), 114-131.

[4] S. Ferretti, M. Furini, C.E. Palazzi, M. Roccetti, P. Salomoni, "WWW Recycling for a Better World", Communications of the ACM, accepted for publication, November 2008.

[5] A. Hars, S. Ou, "Working for Free? - Motivations of Participating in Open Source Projects", in Proceedings of the 34th Hawaii International Conference on System Sciences, Vol. 1, San Diego, USA, January 2001, 286-294.

[6] HR-XML, web site: http://www.hr-xml.org/, last visited December 2008.

[7] The GPX Exchange Format, web site: http://www.topografix.com/gpx.asp. last visited December 2008.

[8] F. Kon, F. Costa, G. Blair, R.H. Campbell, "The case for reflective middleware", Communications of the ACM, 45, 6, June 2002.

[9] A. Nandan, S. Das, B. Zhou, G. Pau, M. Gerla, "Ad-Torrent: Digital billboards for vehicular networks", in Proceedings of the IEEE/ACM International Workshop on Vehicle-to-Vehicle Communications (V2VCOM), Vol. 1, San Diego, USA, July 2005, 286-294.

[10] O. Nov, B. Rao, "Technology-Facilitated 'Give According to Your Abilities, Receive According to Your Needs'". Communications of the ACM 51 (5) 83-87, May 2008.

[11] C.E. Palazzi, S. Ferretti, M. Roccetti, G. Pau \& M. Gerla, "How Do You Quickly Choreograph Inter-Vehicular Communications? A Fast Vehicle-to-Vehicle Multi-Hop Broadcast Algorithm, Explained", in Proceedings of the 4th IEEE Communications and Networking Conference (CCNC 2007), Las Vegas (USA), IEEE, January 2007.

[12] M. Roccetti, S. Ferretti, M. Furini, C. E. Palazzi \& P. Salomoni, "egoisms: What Would the Web be Without the Others?", Proc. 10th ETHICOMP International Conference on the Social and Ethical Impacts of Information and Communication Technology (ETHICOMP2008), Mantua (Italy), September 2008, 678-686.

[13] World Wide Web Consortium, "Composite Capability/Preference Profiles (CC/PP): Structure and Vocabularies 1.0.", Available from: http://www.w3.org/TR/2004/REC-CCPP-struct-vocab-20040115.

[14] Zhu, Y. and Hu, Y. 2007. Efficient semantic search on DHT overlays. $J$. Parallel Distrib. Comput. 67, 5 (May. 2007), 604-616. 